\section{Fabrication of the X-Ray Mask using the Silicon Dry Etching*}

\author{
Hiroshi TSUJII**, Kazuma SHIMADA**, Makoto TANAKA**, \\ Wataru YASHIRO***, Daiji NODA** and Tadashi HATTORI** \\ **Laboratory of Advanced Science and Technology for Industry, University of Hyogo, \\ 3-1-2Koto, Kamigori, Ako, Hyogo, Japan \\ E-mail:thiroishi@lasti.u-hyogo.ac.jp \\ ${ }^{* * *}$ Graduate School of Frontier Sciences, The University of Tokyo \\ 5-1-5Kashiwanoha, Kashiwa, Chiba, Japan
}

\begin{abstract}
The X-ray lithography of uses synchrotron radiation is one of the microprocessing structure fabrication technology. In X-ray lithography, precision of the fabricated structure is influenced by precision of the X-ray mask considerably. Conventionally, the X-ray mask was fabricated with UV lithography. However, it is difficult to fabricate the highly precise X-ray mask because of the tapering X-ray absorber. We introduces the ability of Si dry etching technology into UV lithography in order to fabricate untapered, high precision X-ray masks containing rectangular patterns. This new X-ray mask fabrication method uses a high-precision microstructure pattern formed by Si dry etching, thereby fabricating high aspect ratio, narrow line width resist microstructures that cannot be achieved by any conventional technology. An Au for the X-ray absorber is made to the groove of the structure, and it is formed by electroplating. The silicon substrate itself is used as seed layer and the structure is fabricated with the photo resist whose resistance is higher than silicon. It can be expected the gilding growth from only the bottom layer. High-density Au functions sufficiently as an absorber. Au plating was formed only from the base of the structure ditch and could bury Au of thickness $3.5 \mu \mathrm{m}$ in a narrow place of $2.7 \mu \mathrm{m}$ in width well. The fabricated structure using X-ray lithography. Highly-precise rectangular structure could be fabricated.
\end{abstract}

Key words: X-Ray Lithography, Synchrotron Radiation, X-Ray Mask, Dry Etching, Untapered Structure

\section{Introduction}

Personal computers, cellular phones and other information appliances, as well as TVs, stereos, and other audiovisual equipment have been continually downsized and sophisticated year after year to add to the comfort of users. The parts used in such appliances and equipment has been reduced in weight and energy consumption, and further miniaturization and upgrading of precision parts and components are unavoidable. These requirements can no longer be met by simply improving machining, grinding, and other traditional parts production techniques. Nanotechnology is attracting people's attention as a technology that will play a key role in the miniaturization and sophistication of parts in the future. In particular, Micro electro mechanical systems (MEMS) technology is undergoing remarkable development and contributing to the downsizing and enhancing of parts performance. The MEMS technology is used for fabricating microstructures. X-ray lithography is also used as one of the technologies for fabricating nanometer/micrometer 
size microstructures. X-ray lithography is suitable for fabricating structures at high precision since it uses highly directive a synchrotron radiation ${ }^{(1)}$. For an X-ray lithography process, the accuracy of a fabricated structure depends largely on the accuracy of the X-ray mask used. The reason being that, in an X-ray lithography process, the contrast between the area where the X-ray mask absorber has been formed and the blank area is directly transferred onto the structure being fabricated. This means that the accuracy of the X-ray mask pattern is crucial for X-ray lithography. Therefore, X-ray lithography requires a microfabrication technology that can be employed for fabricating untapered, high aspect ratio X-ray masks. In a conventional X-ray mask fabrication process, a resist structure is first fabricated using UV lithography and then an absorber is formed on the resist structure ${ }^{(2)}$. In an X-ray mask fabrication process that uses only UV lithography, the resist structures are finished with undesired tapering mainly due to the diffraction of ultraviolet when their height is several $\mu \mathrm{m}$. The diffraction of ultraviolet also affects line configuration, making the tapered line more conspicuous as its width decreases. It is therefore extremely difficult to fabricate X-ray masks of highly-precise rectangular patterns using UV lithography only.

Silicon dry etching technology is currently attracting the attention of engineers. This technology uses an inductively coupled plasma (ICP) and is effective for fabricating $\mathrm{Si}$ microstructures. Thus this technology makes it possible to fabricate rectangular structures by etching lines to a depth of $10 \mu \mathrm{m}$ even when their minimum width is as thin as $100 \mathrm{~nm}$.

Our research study introduces the ability of Si dry etching technology into UV lithography in order to fabricate untapered, high precision X-ray masks containing rectangular patterns, which cannot be achieved by UV lithography only. For the study, the $\mathrm{X}$-ray mask to be fabricated was configured as a rectangular line-and-space mask comprising $5.3 \mu \mathrm{m}$ pitch narrow lines. The main body of the text should be suitably divided, i.e., sections and subsections, each with a heading.

\section{Si Dry Etching Technology}

Advanced silicon etching (ASE) is usually used for Si dry etching by an ICP etching system. A conceptual diagram of the ASE process is shown in Fig.1. In the ASE process, a microstructure is etched by repeating two steps alternately. In the etching process, which is a Si reactive ion etching (RIE) process, $\mathrm{SF}_{6}$ gas is introduced into the etching chamber to generate $\mathrm{F}$-radical and $\mathrm{F}$-ion in plasma. In the protective film forming process, $\mathrm{C}_{4} \mathrm{~F}_{8}$ gas is introduced into the chamber to cause a polymerization reaction between $\mathrm{CF}_{\mathrm{X}}$ radical and ion in the atmosphere of plasma, thereby forming a polymer film on the structure surface. The composition of this polymer is almost the same as that of Teflon. Since Si is etched in an anisotropic manner in the RIE process, the protective film formed on the bottom of the structure groove is removed earlier than the film deposited on the side faces ${ }^{(3)}$. Accordingly, it is possible to prevent the etching from progressing in the groove width direction by making the etching rate equal to the protective film formation rate within one etching cycle, thereby etching $\mathrm{Si}$ in the groove depth direction only. In this manner, high aspect ratio microstructures can be fabricated.

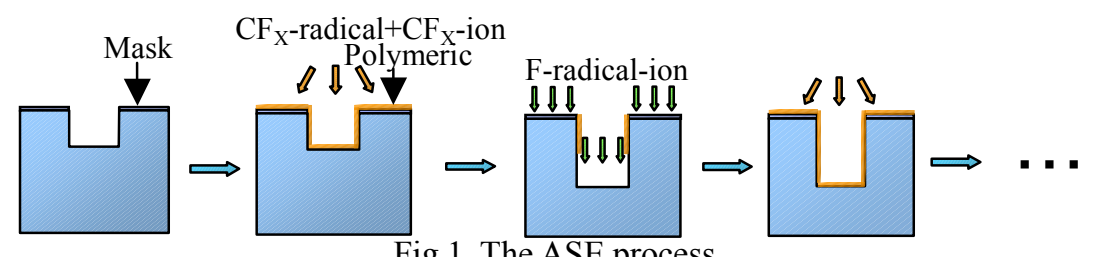

Fig.1. The ASE process 


\section{Conventional X-ray Mask Fabrication Method}

A conventional X-ray mask fabrication method that employs UV lithography is shown in Fig.2. The fabrication process consists of five steps: (a) depositing a seed layer on the substrate, (b) applying a resist over the seed layer to transfer the mask pattern onto the resist using UV lithography, (c) Forming an X-ray absorber on the resist structure that has already been fabricated, (d) forming a membrane on the structure and bonding a frame, and (e) Completing a X-ray mask by etching the substrate. In the X-ray mask fabrication process that employs UV lithography, the resist structures several micrometer high are often tapered due to the diffraction of ultraviolet, as shown in Fig.3. Therefore, it is extremely difficult to fabricate X-ray masks having a high-precision, rectangular pattern by conventional X-ray mask fabrication technology.

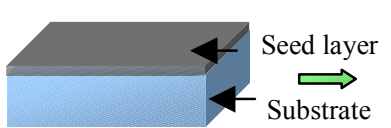

(a)

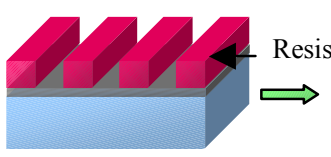

(b)

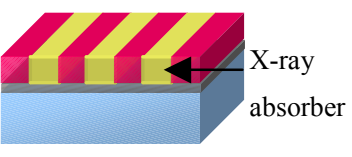

(c)

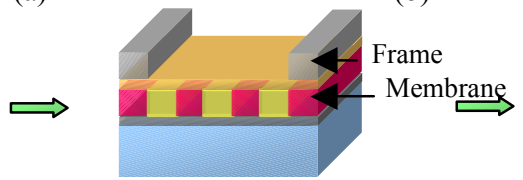

(d)

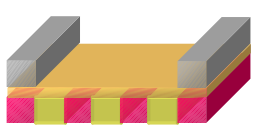

(e)

Fig.2. A conventional X-ray mask fabrication method that employs UV lithography

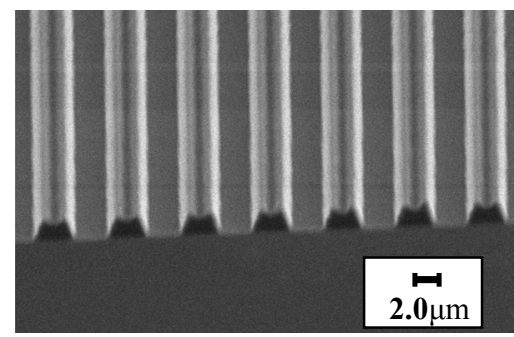

Fig.3. SEM image of the taper structure

\section{Proposal of New X-ray Mask Fabrication Method}

The new X-ray mask fabrication method proposed here is shown in Fig.4. This method consists of 12 steps: (a) depositing $\mathrm{Cr}$ on an $\mathrm{Si}$ substrate by sputtering, $\mathrm{Cr}$ being the etching mask material for Si dry etching, (b) applying a thin film resist, NPR3600PG (made by Nagase chemiteX Corp.), over the substrate with a spinner, (c) patterning on the resist by employing UV lithography, (d) etching the pattern on $\mathrm{Cr}$, using the already patterned resist as a mask material, (e) fabricating an Si microstructure by performing Si dry etching using an ICP etching system, (f) embedding a resist, SU-8 3035 (made by KAYAKU MICROCHEM Corp.) on the Si microstructure groove with a spray coater, then exposing and baking the whole microstructure surface to accelerate the cross-linking reaction of SU-8, (g) irradiating the $\mathrm{Si}$ substrate with $\mathrm{O}_{2}$ plasma to thoroughly remove the resist that has overflowed the Si microstructure groove, (h) removing the Si structure by wet etching, (i) depositing $\mathrm{Au}$ in the groove of the previously fabricated resist microstructure by electrolytic plating, (j) applying a membrane material, SU-8 50 (made by KAYAKU MICROCHEM Corp.), over the microstructure, then exposing and baking the whole surface to accelerate the cross-linking reaction of SU-8 50, (k) bonding a frame with an adhesive to support the X-ray absorber and membrane, and (l) completing an X-ray mask by wet etching the Si substrate.

This new X-ray mask fabrication method uses a high-precision microstructure pattern formed by Si dry etching, thereby fabricating high aspect ratio, narrow line width resist 
microstructures that cannot be achieved by any conventional technology. In conventional fabrication technologies, increasing the thickness of the resist structures leads to the diffraction of ultraviolet when they are subjected to UV lithography. This causes the resist to have a tapered configuration, deflecting it's from a rectangular shape. For high precision microstructure patterns fabricated by Si dry etching, it is difficult to form a seed layer on the bottom of the groove. The seed layer is necessary for electrolytic plating of Au to be used an $\mathrm{X}$-ray absorber. Unless the seed layer is formed on the bottom of the groove only, Au will start growing from both the bottom and side faces of the groove. As a result, voids will generate inside the $\mathrm{Au}$ film. An Au film with voids cannot satisfactorily function as an X-ray absorber. To prevent void generation, we have already proposed to coat the Si substrates with a resist having an electric resistance greater by the order of several digits than $\mathrm{Si}$. This method is expected to accelerate $\mathrm{Au}$ growth from only the bottom of the groove since it provides a large difference in resistance between the bottom and side faces (4). Thus, our new method can form void-free, higher density Au films that will work as high performance X-ray absorbers.

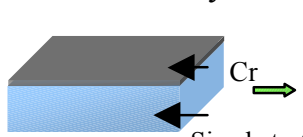

(a)

$\Longrightarrow$

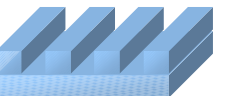

(e)

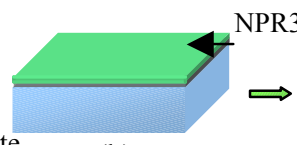

(b)

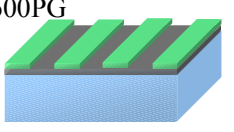

(c)
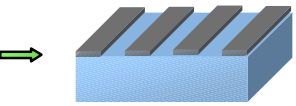

(d)
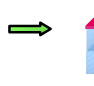

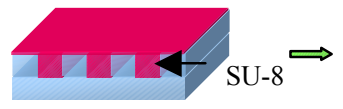

(f)

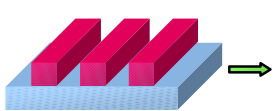

(h)

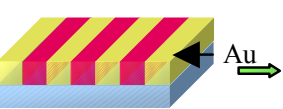

(i)

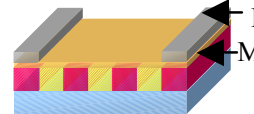

(j)

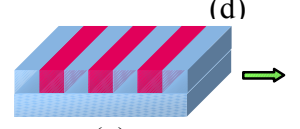

(g) Frame

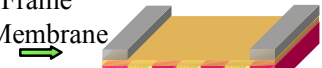

(k)

Fig.4. Proposal of New X-ray Mask Fabrication Method

\subsection{Si Dry Etching by ICP etching system}

$\mathrm{Si}$ microstructures were fabricated on an experimental basis using an ICP etching system apparatus. Preferable microstructures could be fabricated by improving the pretreatment process, such as thoroughly removing $\mathrm{Cr}$, as shown in Fig.5. Microstructures were fabricated under the same conditions as those for Fig.5 but various numbers of etching cycles. As seen from the result shown in Fig.6, Si structures having a width of $1.9 \mu \mathrm{m}$ were obtained and the etching depth increased in proportion to the number of etching cycles. This implies that etching depth could be controlled without changing etching width. The SEM picture shown in Fig. 6 verifies that the newly proposed method was effective for fabricating high aspect ratio, rectangular Si microstructures at a width of $1.9 \mu \mathrm{m}$ with an etching depth of $18 \mu \mathrm{m}$.

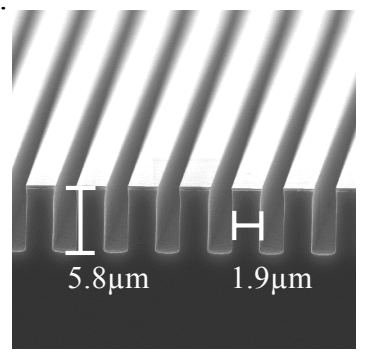

Fig.5. SEM image of preferable structure

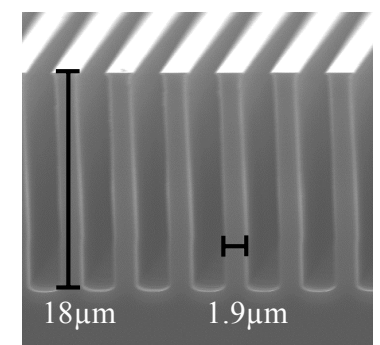

Fig.6. SEM image of high aspect ratio structure

\subsection{Fabrication of Resist Microstructure}

SU-8 was embedded in the groove of a Si structure using a spray coater. The result is shown in Fig.7. The spray coater was effective for embedding a resist in the extreme corners of the narrow groove, which could not be achieved by a spin coater. The resist overflowing 
the Si microstructure groove should be removed until the Si surface is leveled. The resist was removed by irradiating $\mathrm{O}_{2}$ plasma. The substrate after plasma irradiation is shown in Fig.8. It was confirmed from this figure that the resist was thoroughly removed from the whole Si structure surface until it was fully exposed. At the final step, the Si structure body was removed to obtain a high precision, rectangular resist structure.

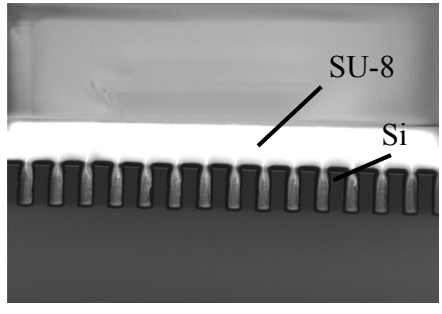

Fig.7. SEM image of embedding a resist on a Si structure

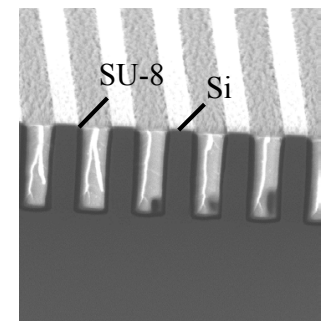

Fig.8. SEM image of the substrate after plasma irradiation

\subsection{Formation of X-ray Absorber}

The Au film to be used as an X-ray absorber was electrolytically plated on the resist structure groove that had been formed in the preceding process. The structure after the Au-plating is shown in Fig.9. As can be seen from the figure, a $3.5 \mu \mathrm{m}$ thick Au film could be formed in a space as narrow as $2.7 \mu \mathrm{m}$. Au did not grow from the side faces of the structure but grew from only the bottom of the structure groove. Thus, a void-free, high density X-ray absorber was successfully formed. For completion as an X-ray mask, the $\mathrm{Si}$ substrate was subjected to wet etching subsequent to membrane formation and frame bonding. The fabricated X-ray mask is shown in Fig.10.

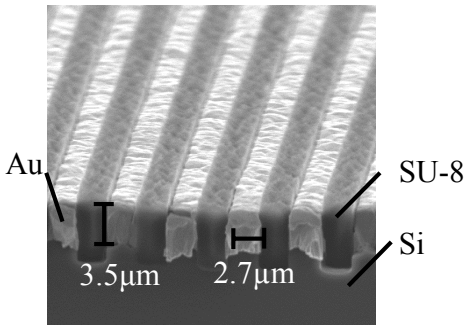

Fig.9. SEM image of the structure after the Au-plating

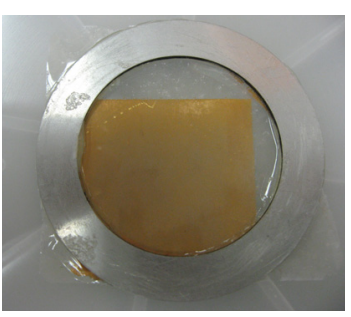

Fig.10.The fabricated X-ray mask

\section{X-ray lithography}

The fabricated structure using X-ray lithography is shown in Fig.11. For X-ray lithography, we used the negative photoresist SU-8. Highly-precise rectangular structure could be fabricated. It could be shown the utility of the X-ray mask fabricated using Si dry etching.

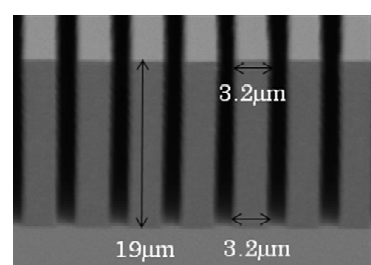

Fig.11.The fabricated structure using X-ray lithography

\section{Conclusion}

X-ray lithography is also used as one of the technologies for fabricating nanometer/micrometer size microstructures. X-ray lithography is suitable for fabricating 
structures at high precision since it uses highly directive a synchrotron radiation. For an $\mathrm{X}$-ray lithography process, the accuracy of a fabricated structure depends largely on the accuracy of the X-ray mask used. This means that the accuracy of the X-ray mask pattern is crucial for X-ray lithography. Therefore, X-ray lithography requires a microfabrication technology that can be employed for fabricating untapered, high aspect ratio X-ray masks. In a conventional X-ray mask fabrication process, a resist structure is first fabricated using UV lithography and then an absorber is formed on the resist structure. In an X-ray mask fabrication process that uses only UV lithography, the resist structures are finished with undesired tapering mainly due to the diffraction of ultraviolet. It is therefore extremely difficult to fabricate X-ray masks of highly-precise rectangular patterns using UV lithography only.

A new method for fabricating high precision, rectangular X-ray masks having a micro-configuration of $5.3 \mu \mathrm{m}$ pitch line-and-space has been proposed in this paper. This method additionally uses Si dry etching in the UV lithography process.

In the present study, a high precision, rectangular resist microstructure was fabricated from a Si microstructure that had been fabricated using an ICP etching system. As a result, it was found that the introduction of the ICP etching system only was not enough for satisfactory Au plating since electric resistance was the same over the whole microstructure surface. To solve this problem, a resist was applied over the Si microstructure surface thus creating a difference in electric resistance. This solution allowed Au to grow from the $\mathrm{Si}$ seed layer on the bottom of the structure groove only. Au was plated on the groove of the resist microstructure that had been fabricated according to the improved method. This resulted in the successful formation of a high density, $3.5 \mu \mathrm{m}$ thick, void-free Au film in a space as narrow as $2.7 \mu \mathrm{m}$. The membrane thickness of the X-ray absorber could be increased up to $3.5 \mu \mathrm{m}$ when a resist microstructure was fabricated by using an approximately $5 \mu \mathrm{m}$ high Si microstructure.

The fabricated structure using X-ray lithography. Highly-precise rectangular structure could be fabricated. It could be shown the utility of the X-ray mask fabricated using Si dry etching.

The study has verified that the new method discussed in this paper is suitable for the high-precision fabrication of finely and complexly patterned X-ray masks, and is expected to be used in the production of a wide variety of devices that have not yet been put into practice.

\section{Acknowledgement}

This research was supported by the research project "Development of System and Technology for Advances Measurement and Analysis" from the Japan Science and Technology Agency (JST).

\section{References}

(1)D. Noda, et al., "Fabrication of Diffraction Grating with High Aspect Ratio Using X-ray Lithography Technique for X-ray Phase Imaging", Japanese Journal of Applied Physics, Vol. 46, No.2, 2007, pp.849-851.

(2)M. Matsumoto, et al., "Fabrication of diffraction grating for X-ray Talbot interferometer", Microsystem Technologies, Vol.13, No.5-6, 2007, pp.543-546.

(3)J. Ohara, et al., "A new deep reactive ion etching process by dual sidewall protectionlayer", MEMS2000, Miyazaki, Japan, 2000, pp277-282.

(4)T. Fujita, et al., "SELECTEVE AND DIRECT GOLD ELECTROPLATING ON SILICON SURFACE FOR MEMS APPLICATIONS”, MEMS2006, Istanbul, Turkey, 2006, pp290-293. 